\title{
Voces indigenas de Tagual La Pó como referencial de una política pública de protección de territorios ${ }^{*}$
}

\author{
Jorge Eduardo Vásquez Santamaría' \\ Fundación Universitaria Autónoma de las Américas (Colombia)
}

Recibido: mayo 15 de 2016 - Revisado: julio 20 de 2016 - Aceptado: septiembre 20 de 2016

Referencia formato APA: Vásquez, J. E. (2016). Voces indígenas de Tagual La Pó como referencial de una política pública de protección de territorios. Rev. Guillermo de Ockham, 14(2), 143-151. doi: http://dx.doi.org/10.21500/22563202.2675

\section{Resumen}

El rescate de las voces indígenas en sus lenguas maternas y en sus lugares de origen, se trazó como el medio ideal para respetar y valorar el pensamiento ancestral como principio esencial de simbolización del territorio, objeto de protección que a partir de las voces indígenas del resguardo Tagual La Pó dio cuerpo al flujo decisional originario que nutre la construcción del referencial de una política pública en el Departamento de Antioquia. Las voces indígenas fueron rescatadas a partir de instrumentos de historia oral que indagaron por las representaciones e interpretaciones sobre el territorio, las relaciones que se entablan con él y las realidades territoriales definidas como problemas públicos que en la actualidad victimizan a los integrantes del resguardo Tagual La Pó e identifican el distanciamiento con las experiencias de comunidades indígenas de las subregiones de Urabá y el suroeste del departamento de Antioquia.

Palabras clave: territorio, voces indígenas, política pública, referencial, flujo decisional.

\section{Indigenous voices from Tagual La Pó as reference to a public policy of land protection}

\section{Abstract}

The rescuing of indigenous voices in their native tongues and in their hometowns, was traced as the means to respect and value ancestral thoughts as a fundamental principle of land symbolization, object of protection from indigenous voices of the shelter Tagual La Pó, gave form to the original decisional flow which nurtures the construction of the referential of a public policy in the department of Antioquia. Indigenous voices were rescued from the oral historical instruments which questioned the representations and interpretations of the land, the relations with it, and the defined territorial realities such as public issues which currently victimize the members of the Tagual La Pó shelter, identifying a distance with the experience of indigenous communities of the sub regions of Urabá and the southeast of Antioquia.

Key words: Territory, indigenous voices, public policy, decisional flow

\footnotetext{
* Resultado de investigación Voces de los pueblos indígenas del departamento de Antioquia como referencial de politica pública para la defensa de los territorios, del Grupo de Investigaciones Orbis Iuris de la Facultad de Derecho de la Fundación Universitaria Autónoma de las Américas, en la línea "Derecho, conflicto e internacionalización”. El autor es investigador principal. Como auxiliares están Patricia A. Barrientos B., Diana Arias, Vanessa Villa y Andrés Felipe Cardona B., estudiantes del programa de Derecho de la Fundación Universitaria Autónoma de las Américas.

1. Abogado y magíster en Derecho de la Universidad de Medellín. Especialista en Derecho Administrativo de la Universidad Pontificia Bolivariana y en Docencia Investigativa Universitaria de la Funlam. Líder del grupo de investigaciones Orbis Iuris de la Facultad de Derecho de la Fundación Universitaria Autónoma de las Américas. E-mail: jorge.vasquez@uam.edu.co
} 


\section{Vozes indígenas do Tagual La Pó como uma referência de uma política pública de proteção dos territórios}

\section{Resumo}

O resgate das vozes indígenas em suas línguas nativas e em suas cidades natais, foi traçada como os meios para respeitar e valorizar o pensamento ancestral como um princípio essencial de simbolização do território, o tema da protecção de vozes indígenas da guarda Tagual La Pó, deu corpo para o fluxo de decisáo que nutre construçáo referencial nativo de uma política pública no Departamento de Antioquia. Vozes indígenas foram resgatados de instrumentos história oral indagado por representaçóes e interpretações do território, as relaçóes estabelecidas com ele, e realidades territoriais definidos como problemas públicos que atualmente vitimam membros da guarda Tagual La Pó, identificando a distância para as experiências das comunidades indígenas nas sub-regióes de Urabá e Sudoeste de Antioquia departamento.

Palavras-chave: Território, vozes indígenas, políticas públicas, referencial, fluxo decisional

\section{Introducción}

La construcción del referencial de una política pública de protección de territorios indígenas del departamento de Antioquia se adscribe a la línea de investigación en "Derecho, conflicto e internacionalización", campo de conocimiento en el que fueron enmarcados tres escenarios concretos de problematización.

En primer lugar, se tiene la conmemoración de los veinte años de la Constitución Política de 1991, acontecimiento que reavivó debates sobre la situación de gran cantidad de materias agendadas por la constituyente como objeto de desarrollo legislativo, acciones administrativas y protección judicial. La celebración del segundo aniversario de la norma suprema que representa el pacto colectivo del Estado a través del cual se definieron los valores y fines para la protección y garantía de los derechos humanos y en el cual el individuo es el fin del ordenamiento jurídico y de la acción estatal, han motivado la indagación de aquellas esferas de la realidad social más violadas y vulnerables que, justamente, resultan como pilares del Estado social de derecho. En nuestro caso, se indaga a los pueblos indígenas con los cuales fuera de tener una deuda histórica no han resultado ajenos a las consecuencias del conflicto armado y no armado, puesto que han sido forzosamente desplazados de sus territorios tanto por grupos al margen de la ley como por fuerzas del Estado y multinacionales, lo que ha desencadenado la pérdida del territorio, y con ello de todo su acervo cultural, histórico, social e identi- tario ancestral a partir del cual se teje la cosmovisión del colectivo.

El segundo contexto de problematización recae sobre los esfuerzos y el activismo de los pueblos indígenas en el marco del segundo decenio internacional de los pueblos indígenas del mundo que se conmemoró entre el 2005 y el $2014^{2}$ por declaración de la ONU, luego de un primer decenio entre 1995 y 2004 y declararse 1993 como el año internacional de las poblaciones indígenas del mundo (Organización de las Naciones Unidas, 2013).

Finalmente, el tercer escenario se define a partir de las conversaciones entre los actores del conflicto armado interno llevadas a cabo en La Habana, Cuba, de las cuales se desprende una de las manifestaciones más claras y delicadas de la victimización de las comunidades indígenas. Homicidios, torturas, desplazamientos forzados, reclutamiento de menores y violencia sexual contra mujeres, son algunos de las desgracias que diversas comunidades indígenas padecieron a manos de los actores del conflicto. Estas realidades se suman a las ya existentes, que hacen de las comunidades indígenas una minoría de atención prioritaria.

De acuerdo con Acnur, dieciocho pueblos indígenas están en peligro de desaparecer de los apenas ochenta y siete que han sobrevivido a la colonización (Acnur, 2012). Conforme lo señalado por la Organización Nacional Indígena de Colombia (ONIC), treinta y dos pueblos in-

2. El fomento de la no discriminación y de la inclusión de los pueblos indígenas en la elaboración, aplicación y evaluación de la legislación, las políticas, los recursos, los programas y los proyectos en todos los niveles; el fomento de la participación plena y efectiva de los pueblos indígenas en las decisiones que afectan directa o indirectamente a sus estilos de vida, a sus tierras tradicionales, su integridad cultural o cualquier otro aspecto de sus vidas; la redefinición de las políticas de desarrollo para que incluyan una visión de equidad y de la diversidad cultural y lingüística de los pueblos indígenas; la adopción de políticas, programas, proyectos y presupuestos que tengan objetivos específicos para el desarrollo de los pueblos indígenas, con parámetros concretos e insistiendo en particular en las mujeres, los niños y los jóvenes indígenas; la creación de mecanismos de supervisión estrictos y la mejora de la rendición de cuentas a todos los niveles en lo tocante a la aplicación de los marcos jurídicos, normativos y operacionales para la protección de los pueblos indígenas y el mejoramiento de sus vidas. 
dígenas presentan una situación especial de los ciento dos existentes, toda vez que cuentan con menos de quinientas personas. Según la Corte Constitucional, son treinta y cinco grupos los que están en peligro de extinción a causa del conflicto y del desplazamiento (auto 004 de 2009 y auto 382 de 2010).

El peligro de desaparición de los pueblos indígenas -reconocido por el poder judicial- contrasta con las garantías por él promulgadas con base en la norma fundamental. ${ }^{3}$ Disposiciones como los artículos 8, 9, 10, 63,329 y 330 se conjugan en un entramado de tensiones con figuras colectivas como el territorio, el ambiente y las políticas públicas, sin dejar de lado las obligaciones que se desprenden de normas como el Convenio sobre Pueblos Indígenas y Tribales en Países Independientes, de la Organización Internacional del Trabajo (OIT) y la Declaración Universal sobre los Derechos de los Pueblos Indígenas, de la Organización de las Naciones Unidas. ${ }^{4}$

La desidia del Estado colombiano devela la ausencia de diligencia para poner en funcionamiento instituciones y políticas a partir de normativas de distinta naturaleza que no solo contengan, sino que también respeten el pensamiento ancestral de estos colectivos minoritarios. A ello se suma el auge de los proyectos mineros y de infraestructura para la prestación de servicios que amenazan los territorios ancestrales y su autonomía jurisdiccional y la segregación en los contextos urbanos. Si bien el Estado no resuelve de manera concreta su indiferencia ante la extinción de los pueblos indígenas, la sociedad civil no muestra tampoco un interés real en hacer notar que esta población vulnerable está siendo desatendida, tal vez porque aún se sigue considerando como un pueblo sin civilización ni cultura o simplemente porque son diferentes. Ello acrecienta la brecha social y aumenta la legitimidad de un mero poder simbólico de lo jurídico, en contrapeso a la ineficacia en la realización de derechos.
[...] el $63 \%$ de la población indígena vive bajo la línea de pobreza y el 47,6\% no tiene los ingresos para adquirir una canasta de alimentos que cubra los requerimientos alimenticios mínimos diarios (línea de miseria). El 34,53 \% no tiene acceso a la salud. La tasa de analfabetismo entre los quince y los cuarenta y nueve años es del $24,1 \%$, mientras la de la población mestiza es seis veces menor y solamente el $50 \%$ entre los cinco y los veinticinco ańos asiste a alguna institución educativa. De otra parte, tres de cada cinco indígenas no tienen acceso a acueducto, cuatro de cada cinco no tienen acceso a alcantarillado y dos de cada cinco no tienen acceso a energía. La población indígena tiene una cobertura en acueducto cuatro veces menor a la de los mestizos, 3.2 veces menor en alcantarillado y 9.3 veces menor en servicio de energía (Justicia Global y Derechos Humanos de la Universidad de los Andes, 2009 citado por Konrad, 2012).

Si bien la realidad indígena debería ser reconocida como común u ordinaria, en un contexto como el colombiano no es así. La territorialización se define mayoritariamente en términos jurídico-políticos de desarrollo, generando con ello la perpetuación del choque entre los esfuerzos por la defensa de los territorios y las imposiciones jurídicas y políticas del Estado. De ello se desprenden importantes bienes jurídicos que dan cuenta de la construcción de una acepción no indígena sobre el territorio, como recurso que no se corresponde con políticas en las que los pueblos indígenas mantienen su voz de lucha y su invitación a la movilización por la reivindicación de su inclusión y autonomía.

La realidad de los pueblos indígenas debería ser un eje común en un sistema jurídico liderado por una Constitución adoptiva del Estado social de derecho, en un contexto político de diálogos para la superación de la confrontación armada del conflicto interno, a partir del cual se promueva tanto legislación garantista (promotora de acciones afirmativas) que les reconozca su naturaleza de personas de especial protección, como la obligación de

3. Véanse las sentencias T-001 de 2012, T-129 de 2011, T-282 de 2011 C-139 de 1996, C-694 de 2003, T-769 de 2009, C-139 de 1996 y T-496 de 1996.

4. El peligro de desaparición de los pueblos indígenas resulta más problemático cuando se enmarca en la situación del territorio. Los territorios son necesarios para que los indígenas desarrollen sus formas de vida en comunicación con las tradiciones que en ellos se hacen lugares comunes para constituir la identidad. Cuando no se tiene un lugar propio es difícil sentirse autónomo y libre. En este sentido, el artículo 13 de la Declaración Universal sobre los Derechos de los Pueblos Indígenas queda sin fuerza cuando afirma: "1. Los pueblos indígenas tienen derecho a revitalizar, utilizar, fomentar y transmitir a las generaciones futuras sus historias, idiomas, tradiciones orales, filosofías, sistemas de escritura y literaturas, y a atribuir nombres a sus comunidades, lugares y personas, así como a mantenerlos". El artículo 25, por su parte, afirma que los pueblos indígenas "tienen derecho a mantener y fortalecer su propia relación espiritual con las tierras, territorios, aguas, mares costeros y otros recursos que tradicionalmente han poseído u ocupado y utilizado y a asumir las responsabilidades que a ese respecto les incumben para con las generaciones venideras". El artículo 26 reza: " 1 . Los pueblos indígenas tienen derecho a las tierras, territorios y recursos que tradicionalmente han poseído, ocupado o utilizado o adquirido. 2. Los pueblos indígenas tienen derecho a poseer, utilizar, desarrollar y controlar las tierras, territorios y recursos que poseen en razón de la propiedad tradicional u otro tipo tradicional de ocupación o utilización, así como aquellos que hayan adquirido de otra forma". En muchos casos, dicha propiedad no es solemnizada con suficiencia por el Estado, a lo que se suman las debilidades de las políticas y estrategias necesarias para garantizarles la autonomía que dicha propiedad otorga y la falta de títulos sobre sus tierras, lo que afecta el derecho real a oponerse a que en ellas se den combates entre actores armados o se hagan explotaciones mineras. 
recibir el legado y elaboración indígenas para la construcción del Estado nación.

El objeto de estudio de esta investigación se concentra en los pueblos indígenas asentados en el departamento de Antioquia, toda vez que en él habitan 28.000 individuos de tres grandes familias: los emberá, los tule y los zenúes (Medellín, Portal Educativo, 2014) cifra similar a la ofrecida por la gobernación de Antioquia que el censo de 2010, sin contar la población asentada en el área metropolitana del Valle de Aburrá, fijó que en las seis subregiones las comunidades son: Urabá (11.908), occidente (7.106), suroeste (4.266), Bajo Cauca (6.295), nordeste (567) y Magdalena Medio (68) (Gobernación de Antioquia, 2010).

Del total de 30.210 indígenas, 28.192 viven en comunidades del área rural. Los que cuentan con la organización del cabildo, pertenecen en su mayoría al grupo étnico emberá con 20.383 habitantes. Le sigue el grupo étnico zenú con 8.560 habitantes y con menor población está la comunidad tule (kuna) con 1.267 habitantes.

Los municipios con mayor población indígena son, en su orden: 1. Dabeiba, con 4.206 pobladores indígenas; 2. Frontino con 2.802; 3. Turbo con 2.067; 4. Necoclí con 1.984; 5. Chigorodó con 1.885; 6. Jardín con 1.740; 7. Murindó con 1589; y 8. Zaragoza con 1426 (Gobernación de Antioquia, 2010).

Los pueblos indígenas, además de ser una minoría poblacional de especial protección a nivel nacional, son también una minoría amenazada en Antioquia, en especial el resguardo Tagual La Pó, en el nordeste del departamento. Sus integrantes también son víctimas históricas recurrentes de conflictos, especialmente el armado que atenta contra sus territorios como patrimonio del modelo de vida. Situaciones como la que representa Tagual La Pó exigen superar la indagación de directrices normativas como primordiales referentes nominales de reconocimiento y protección de derechos y en contraposición, promover en perspectiva sociojurídica la relación de esos pueblos con el territorio a partir de la carga simbólica expresada por medio de sus voces en lengua materna, las cuales han sido excluidas o aminoradas por la influencia de los conflictos y el ejercicio del poder, en un ámbito constitucional en el que resultan ser una institución fundamental para la construcción de la democracia. En ese orden, se hace pertinente revivir la relación entre los pueblos indígenas y el territorio como bien jurídico que se debe proteger, mediante el rescate de flujos de decisión que construyan referentes para políticas públicas que respondan a las necesidades territoriales reales de estos pueblos. La pregunta de investigación que orientó este trabajo fue la siguiente: ¿En qué sentidos las voces de los pueblos indígenas del departamento de Antioquia constituyen un referencial de política pública para la defensa del territorio? A partir este interrogante se presenta el rescate del flujo decisional de Tagual La Pó, componente que contribuye a la construcción del referencial de política pública de protección de territorios.

\section{Método}

De acuerdo con la naturaleza sociojurídica del objeto de estudio inscrito en un campo científico de tipo interdisciplinar, el diseño metodológico propuesto parte del modelo de investigación cualitativo acompañado del enfoque histórico hermenéutico, trabajado a partir del territorio, la política pública, el flujo decisional y el referencial de política como categorías predefinidas principales.

El territorio se aborda, en este caso, como un concepto cultural (Zapata, 2010), toda vez que es la materia a partir de la cual se define el orden simbólico que hace posible el desarrollo comunal de los colectivos indígenas y posibilita la permanencia y la transmisión de sus tradiciones, pensamientos, anhelos y necesidades (Poloche, 2012). El territorio, más que categoría es categorizador cultural, "signo cuyo significado se construye desde dos lugares: desde los códigos socioculturales en los que se inscribe y desde los códigos de quienes lo interpretan" (Rincón \& Echavarría, 2000, p. 25). De ahí que: "para los pueblos indígenas de Antioquia, el territorio es centro de desarrollo cultural y cosmogónico y no debe convertirse en escenario de guerra o conflicto" (ONIC, 2010). Para la categoría de política pública se privilegió la elaboración de Muller (2002) quien la explica cómo "un proceso de mediación social, en la medida en que el objeto de cada política pública es tomar a cargo los desajustes que pueden ocurrir entre un sector y otros, o aun, entre un sector y la sociedad global”. Señala que su enfoque es una construcción de representaciones sociales de la acción pública y de las relaciones entre el Estado y la sociedad, para luego agregar el concepto de referencial de una política, concebida como el conjunto de normas, imágenes y expectativas que posibilitan o restringen el funcionamiento del sistema político y del régimen político para solucionar los problemas sociales. Esta figura se edifica a partir de la confluencia de flujos decisionales, entendidos en nuestro caso como ejercicios de participación democrática activa en los cuales los sujetos asumen el papel de actores políticos en dinámicas que posibilitan la gobernanza y se involucran en términos de formulación de política, pero también de proposición de 
alternativas, ejecución de ellas y evaluación para revitalizar posteriores formulaciones.

Según el citado contexto metodológico y teórico se hicieron entrevistas y grupos focales como instrumentos de oralidad que permitieron rescatar el flujo decisional. La implementación de los instrumentos de historia oral se organizó en un trabajo de campo con seis comunidades indígenas de las subregiones de Urabá, suroeste y nordeste de Antioquia a partir del segundo semestre de 2014 hasta el segundo semestre de 2015. En esas zonas se emprendió la construcción del referencial de política pública articulada al enfoque de ciclo, lo que dio lugar a la descripción de las problemáticas y a su interpretación a partir de las narrativas, testimonios e historia oral obtenidos en los resguardos de El Volao en el municipio de Necoclí, y las comunidades Las Playas y La Palma, distribuidas en los municipios de Turbo y Apartadó, todas de la subregión de Urabá, así como la comunidad La María o Marcelino Tascón en el municipio de Valparaíso y la comunidad karmata rua, distribuidas entre los municipios de Andes y Jardín, ambas de la subregión suroeste y con la comunidad Tagual La Pó, del municipio de Segovia en el nordeste de Antioquia.

Los instrumentos de historia oral empleados se guiaron por un conjunto de preguntas organizadas en tres bloques. Primero, se trabajó a partir de interrogantes relacionados con el territorio: ¿qué representa para ustedes el territorio? ¿Cómo se relacionan ustedes con el territorio? ¿Cuál es la relación que más desean mantener con el territorio? Un segundo momento se centralizó en la siguiente pregunta: a partir de su lenguaje, sus diálogos y sus narrativas, ¿cómo explican el territorio en su comunidad? El bloque más amplio se concentró en los problemas vinculados con el territorio e inicialmente se proponen preguntas a partir de los hallazgos en investigaciones tomadas como precedentes: ¿su territorio presenta problemas ambientales? ¿Presenta problemas de prestación y atención en salud? ¿Problemas en la prestación y calidad de la educación? ¿Presenta problemas por el conflicto armado? Luego de indagar por las dificultades identificadas en investigaciones precedentes como ejercicio de validación de las realidades encontradas en las fuentes documentales, se propusieron preguntas de ampliación y reconstrucción de los problemas actuales: ¿en su territorio tienen ustedes actualmente otro tipo de problemas? ¿Cuál es el problema que consideran es el más grave? ¿Qué alternativas proponen para darle solución a ese problema? ¿Cómo comunican la existencia de este tipo de problemas a las autoridades del Estado?
Este escrito presenta parte del referencial de política pública construido a partir de las voces indígenas de la comunidad Tagual La Pó a través de su gobernador actual, el exgobernador, uno de los maestros y tres líderes de la comunidad, sin que fuera posible contar con participación de mujeres. Con el referencial se describen las realidades sociojurídicas que los excluyen y victimizan, se exponen las interpretaciones sobre de las representaciones del territorio elaboradas por la comunidad y se evidencian formas a partir de la cuales se relacionan con él. La construcción del referencial se apoya en el reglamento interno del resguardo, fuente de información de construcción propia de la comunidad y un referencial normativo para la política pública desde el cual se ponderan y valoran los insumos orales que resultan de las voces indígenas que permitieron rescatar el flujo decisional.

\section{Representaciones e interpretaciones del territorio en Tagual La Pó}

De acuerdo con la tendencia de las comunidades de la subregión de Urabá y del suroeste de Antioquia, las voces de Tagual La Pó exponen el significado del territorio desde un enfoque materno de sobrevivencia, identidad y localización con el mundo y para él, exaltando algunas de las razones que lo reafirman como objeto colectivo de protección. En palabras del gobernador: "Territorio es donde ocupan los indígenas, una comunidad indígena, es territorio donde se ocupa, y es territorio indígena un territorio colectivo de la comunidad indígena." (EGTP, 6 de diciembre de 2015), representación coincidente con la del exgobernador, quien expuso: "Territorio para nosotros significa, es, donde vivimos todos los indígenas, donde trabajamos, donde hacemos casa, donde están las quebradas, donde están los bosques, donde están todas las supervivencias de los indígenas" (EEGTP, 6 de diciembre de 2015).

Los testimonios de las autoridades del resguardo fueron refrendados tanto por el maestro: "Territorio hace parte de la vida cotidiana de los pueblos indígenas, porque allí vivimos, allí nacimos y todo lo que ella produce es para nosotros" (EMTP, 6 de diciembre de 2015), como por uno de sus líderes: "Territorio es donde nosotros nos resguardamos, donde viven nuestras familias y es lo que debemos cuidar" (EL1TP, 6 de diciembre de 2015).

Si bien las voces indígenas de Tagual La Pó resultan coincidentes con las representaciones de las demás comunidades indagadas en Antioquia, la interpretación que se potencializa sobre el sentido del territorio para una política pública se identifica en los referentes expresados 
sobre las formas de relación con el territorio, así como la selección de aquella forma que desean mantener como relación debida. Sobre la relación de las comunidades con el territorio, se tiene lo siguiente:

Gobernador: "la relación, a pues, el territorio primera parte es colectiva, ocupada por la comunidad indígena y tiene su reglamento; habitan 419 indígenas, 188 menores" (EGTP, 6 de diciembre de 2015).

Exgobernador: "Nos relacionamos con el territorio, principalmente bregar a cuidarlo y proteger muchas cosas, de la naturaleza, proteger la biodiversidad que hay entre las montañas las plantas, las creencias, las culturas todo eso, hay que proteger mucho" (EEGTP, 6 de diciembre de 2015).

Líder 1: "El territorio es lo mejor que puede tener el ser humano porque es lo que nos da el comer de nuestra vida" (EL1TP, 6 de diciembre de 2015).

A diferencia de las pretensiones de participación, cumplimiento de políticas, restauración ambiental y conservación de las costumbres y prácticas ancestrales que sobresalen en las comunidades de Urabá y suroeste, en el caso del nordeste emergen con Tagual La Pó relaciones con el territorio caracterizadas por la demanda de grandes esfuerzos, luchas y desgastes para los indígenas. Lo anterior se deja leer en las representaciones de conservación y respeto por la tierra, en las dificultades de los dirigentes indígenas frente a la manutención de los recursos y sobre todo en el claro control de información que se manifestó en casi todas las entrevistas, en las cuales se reiteraron el número de habitantes y su crecimiento en el tiempo, la fecha de constitución del resguardo y la clarificación de datos que definen su identidad, aspectos que abren la puerta a las problemáticas públicas actuales de este resguardo y siembran la idea del temor por la desaparición. La diferenciación que define las representaciones y relaciones con el territorio en Tagual La Pó se acentúa a partir de la referenciación de la relación anhelada con dicho recurso. Ante la pregunta acerca de la relación que mas desearían mantener con el territorio, se obtuvieron las siguientes respuestas:

Exgobernador: "Lo que más desea uno, quiere mantener con el territorio ojalá tener buen trabajo, buena administración, proyectos productivos, proyectos que sostienen" (EEGTP, 6 de diciembre de 2015).
Maestro: "Que no se les quite más el lugar, el espacio, que les sea permitido expandirse, en todo lo relacionado con el lugar donde habitan [...] que ningún extranjero venga a dañar el seno de la tierra porque nos puede perjudicar" (EMTP, 6 de diciembre de 2015).

De las voces indígenas emergió tanto una representación del territorio como una relación con el mismo basada en la presencia de amenazas, evidentes y actuales, la vulnerabilidad frente a los proyectos mineros y la llegada de foráneos e inversionistas que retan directa e indirectamente al resguardo indígena.

La ubicación de esta población en el departamento de Antioquia es estratégica. El municipio de Segovia es una jurisdicción definida en los últimos años por la presencia de un conflicto abierto con duros momentos de recrudecimiento, debido principalmente a la riqueza del subsuelo y a los intereses estatales, paraestatales y extranjeros ${ }^{5}$ sobre la explotación de los recursos minerales. En medio de esta realidad, el resguardo Tagual La Pó requiere un trato diferencial por parte de la agenda pública, a lo cual se añade que se trata de una comunidad indígena que fácilmente encaja en las treinta y dos definidas en riesgo por la Corte Constitucional, la ONIC y la Acnur.

La preocupante situación de Tagual La Pó se sustenta a partir de condiciones internas y propias del resguardo. $\mathrm{Si}$ bien no se cuenta con la fecha de registro del reglamento interno, de él se desprende como fecha de constitución el 24 de mayo de 1996, con apenas 374 habitantes, cifra que en el registro de la gobernación de Antioquia de 2004 se ubicó en veinticinco familias que sumaban 268 personas, pero que según datos de la gerencia de asuntos indígenas de la misma gobernación, en el 2013 el resguardo contaba con un total de 472 habitantes, distribuidos de la siguiente manera: 46 en el asentamiento La China; 29 en Urudrua, y 397 en Tagual La Pó (Gobernación de Antioquia, 2015).

La escasez de población debe asociarse con las dificultades para la defensa y mantenimiento de un territorio altamente codiciado por agentes externos. La primacía que redunda en las representaciones sobre el valorado bien colectivo consta en el reglamento interno del resguardo, al cual atribuyen una tenencia desde 1940, lo que define a los indígenas como originarios en un vínculo ancestral y cultural que justifica la más alta jerarquía de las normas creadas por el resguardo en el reglamento interno:

5. Una aproximación a la conflictiva realidad minera del municipio de Segovia se puede apreciar en el texto "Conflicto y colaboración en la minería de oro en Segovia y Remedios”, de Jorge Eduardo Cock y Wilfredo López, en el libro Aglomeraciones Mineras y Desarrollo Local en América Latina, compilado por Rudols M. Buitelar (2001). El Informe Especial "Minería, conflictos sociales y violación de derechos humanos en Colombia", del Centro de Investigación y Educación Popular - CINEP, programa por la paz (2012) 
Artículo 1. Derecho mayor. Este reglamento será regido bajo el permiso de los espíritus y conocimientos ancestrales de nuestros hermanos mayores que de generación en generación nos han trasmitido y nosotros como sus hijos hemos venido recorriendo sus caminos, en sus tradiciones, usos, costumbres, diversos pensamientos y sentires como pueblos originarios, donde lo más fundamental es el respeto a la vida, a la lucha constante de la resistencia en defensa de nuestros territorios, a la defensa de la autonomía, la paz y la armonía con la madre tierra y todos los seres que en ella habitan.

El reconocimiento de las normas del resguardo y los cometidos axiológicos fijados en el artículo primero se acompañan de los principios de unidad (artículo 2) derechos humanos (Artículo 3), responsabilidad (artículo 4) y honestidad (artículo 5), derroteros a partir de los cuales se orienta el funcionamiento de las comunidades del resguardo y se motiva su acción para la defensa del territorio.

\section{Los problemas públicos territoriales de Tagual La Pó}

Mientras en los resguardos de Urabá y del suroeste antioqueño las dificultades de expansión de las jurisdicciones resultaron ser el principal problema público acual para una política dirigida a los indígenas, en Tagual La Pó contextos como el ambiental, el educativo y de salud figuraron como realidades complejas que aquejan a la población, al mismo tiempo que el conflicto armado interno cobró vigencia en las voces de los indagados, fenómeno no conocido por comunidades del suroeste y definido como pasado por las de Urabá.

\section{Problemas ambientales y de salud}

La minería reaparece al preguntar por los problemas ambientales del territorio. Según el gobernador:

es un problema ambiental, pues por aquí por cuestiones de minería, la minería; eso sí pues, sobre cuestiones de depures (sic) de tierra, muchas veces la talación (sic) también, eso son los dos puntos que se ven aquí. Cuando hay mina ya la quebrada se trae contaminada.

La realidad ambiental descrita por el testimonio del gobernador toca directamente con los problemas de salud del resguardo:

Nosotros últimamente tenemos acueducto; lo entregaron el 7 de octubre de este año (2015). Antes el agua era cogida así de la quebrada, recogida en manguera y la arrimaban a las casas así cruda, natural (EGTP, 6 de diciembre de 2015).

En la entrevista con el exgobernador del resguardo y el maestro la tendencia fue idéntica:
Hay problemas ambientales con la naturaleza, la madera, la minería, el yacimiento de agua, todo eso, hay varias cosas que... no sé." En territorio el más importante es el agua (EEGTP, 6 de diciembre de 2015).

Todo es por la tala de árboles, porque cuando yo llegue acá en el 2003 eso es impresionante la tala de árbol por el comercio que ha tenido en este momento hay madera hasta que no se puede, ni sirve para construir casa. La minería es lo mismo. No hay forma de que puedan sostener a la familia, el cultivo es muy poquito y sí lo hacen, pero de todas formas corren detrás de las minas porque es el sustento que hay en estos momentos. Entonces lo que lleva a uno a pensar en cuestión de territorio que hay que cultivar, trabajar, porque lo hemos dicho a ellos que no simplemente vamos a vivir de la minería, sino que hay que tener otra visión distinta (EMTP, 6 de diciembre de 2015).

\section{Problemas educativos}

El problema de falta de educación parece no dar tregua ni ofrecer un porvenir diferente en manos de la propia comunidad como actora empoderada para emprender el cambio sobre problemas interconectados entre sí. La realidad de la educación se define por la falta de recursos. $\mathrm{Al}$ respecto manifestó el Gobernador: No tenemos escuela propia; nosotros mismos nos enseñamos" (EGTP, 6 de diciembre de 2015). En palabras del maestro, la realidad educativa adquiere una definición más completa y similar a la expresada por los resguardos de Urabá y Suroeste:

A nivel educativo manejamos el mundo de dos formas: el mundo emberá y el mundo de Occidente. Entonces nos toca hacer. Dictamos también lo de nosotros si dictamos lo de afuera entonces. Nuestra lengua emberá chamil también la enseñamos; y lo de espańol. Entonces, hacemos las dos cosas al mismo tiempo. Se enseñan dos idiomas porque el emberá es de la familia, donde viven hablan mucho el emberá. Pero, entonces, ¿qué pasa? Nos metieron otro idioma según la ley, una tercera lengua que es el inglés. También estamos manejando los niños con ese inglés (EMTP, 6 de diciembre de 2015).

Debe destacarse que el reglamento interno del resguardo prevé como responsabilidad del cabildo resolver los problemas ambientales, territoriales, de educación y salud (artículo 15), además de apoyar a los estudiantes según las necesidades educativas (artículo 15). La educación de los menores de edad está prevista como una obligación en proporción igualitaria para las parejas que se separen (artículo 36), además de prever acciones para promover la educación encaminada a evitar enfermedades sexuales (artículo 43) y reconocerla como el medio a través del cual se responsabiliza a los padres con el cuidado y estado de salud de sus hijos (artículo 47). Las disposiciones 
anteriores se unen a las previstas en el capítulo $\mathrm{V}$, artículos 58 a 62, que reglamentan el perfil docente para el resguardo, el incumplimiento de sus funciones, el abuso y mal ejemplo a sus alumnos, los actos de desobediencia hacia las autoridades indígenas y la violación y abuso de docente a estudiante.

\section{La presencia de la guerra en un territorio de oro}

Tagual La Pó es un resguardo indígena que padece los avatares del conflicto armado interno de Colombia. Ese temible problema público que parecía superado en la construcción del referencial en las comunidades de Urabá y el suroeste reposiciona las amenazas del conflicto a los territorios indígenas en Segovia:

Esta comunidad ha sido muy sufrida, lo único que yo tengo para decirle es que esta comunidad es de resistencia, no tengo más para decir." (EEGTP, 6 de diciembre de 2015).

Si eso se ha presentado continuo, continuo porque siempre los grupos armados se han trajinado en nuestro territorio y también lo han violado, nuestros derechos, ósea, la regla de la leyes indígenas la han violado y eso sea frenteado aquí (Gobernador. EGTP, 6 de diciembre de 2015).

El conflicto armado en el 2003 era fuerte, siempre se ha presentado, en este momento está casi muy controlado porque los líderes hemos tenido capacidad de decir a ellos que en el resguardo no pueden quedarse más de cinco minutos, pasen no que no pueden pasar, que pasen, pero que no se demoren, en este momento está muy controlado pero el conflicto armado siempre nos han reventado mucho (Maestro. EMTP, 6 de diciembre de 2015).

La presencia del conflicto se refleja desde 1996 en el reglamento interno. Junto con las disposiciones sobre amenazas (artículo 70), daño a los bienes y cultivos de los comuneros o su hurto (artículos 71 y 72 ) y porte de armas dentro de la comunidad (artículo 73), se lee:

Cuando un joven una joven es reclutado en contra de su voluntad, el cabildo, su familia, con el apoyo del consejo de gobierno de la OIA pueden solicitar al grupo armado la liberación del joven o la joven. Cuando un comunero o comunera se une a las filas de un grupo armado ilegal de manera voluntaria, se le solicitará que se retire de la comunidad para evitar riesgos para su familia y a la comunidad (artículo 69).

\section{Conclusiones}

La construcción del referencial de una política pública de protección de territorios indígenas en Antioquia encuentra como uno de sus potenciales el flujo decisional, consolidado a partir de la priorización de la historia oral como un determinante desde el cual se recrean las problemáticas públicas de grupos poblacionales minoritarios, insumo que debe promover un nuevo ciclo en la agenda pública de las autoridades competentes del departamento de Antioquia.

Las voces de los pueblos indígenas del resguardo Tagual La Pó dan lugar a un referencial que altera los consensos identificados en las subregiones de Urabá y suroeste de Antioquia. El enfoque diferencial que exigen los indígenas se debe particularizar y especializar aún más en el caso del resguardo del municipio de Segovia, donde los problemas ambientales, de salud y educación siguen vigentes y concurren con los derivados del conflicto armado interno. Las premuras sobre el territorio colectivo se agravan por variables relacionadas con la explotación minera, lo que devela la construcción de un referencial en el cual la postura de las autoridades del Estado se caracteriza por la falta de presencia, debilidad en las acciones emprendidas por el reconocimiento, protección y defensa de una comunidad que no logra superar el umbral de los 500 habitantes y la desigualdad respecto a las comunidades de las otras subregiones en el respaldo a proyectos de la comunidad.

\section{Referencias}

Corte Constitucional. República de Colombia. (1998). Sentencia $S U$ - 747. Recuperado de https://goo.gl/2ANi0s

Corte Constitucional. República de Colombia. (2009) Auto 004. Recuperado de https://goo.gl/XdcwXW

Corte Constitucional. República de Colombia. (2010) Auto 382. Recuperado de https://goo.gl/dl45xK

EEGTP. (2015). Entrevista ex Gobernador Tagual La Pó 6 de diciembre de 2015.

EGTP. (2015). Entrevista Gobernador Tagual La 6 de diciembre de 2015.

EL1TP. (2015). Entrevista Lider 1 Tagual La Pó 6 de diciembre de 2015.

EMTP. (2015). Entrevista Maestro Tagual La Pó 6 de diciembre de 2015.

Gerencia Indígena de la Gobernación de Antioquia. (2015) Respuesta derecho de petición Nro. R 201400432957 de septiembre de 2014.

Gobernación de Antioquia. (2010). Censo consolidado de las comunidades indigenas en Antioquia 2010. Recuperado de https://goo.gl/RRwI6L

Konrad, A. S. (2009) Situación de los pueblos indigenas de Colombia. Recuperado de http://www.kas.de/wf/doc/ kas_17956-544-4-30.pdf 
Muller, P. (2002). Las políticas publicas. Bogotá: Universidad externado de Colombia.

ONIC-Organización Nacional Indígena de Colombia. (2010) Palabra dulce, aire de vida: Forjando caminos para la pervivencia de los pueblos indigenas en riesgo de extinción en Colombia. Recuperado de www.onic.org.co

ONU-Organización de las Naciones Unidas. (2013). Segundo Decenio Internacional de los Pueblos Indígenas del Mundo (2005-2014). Recuperado de https://goo.gl/pyEM97
Poloche, N. (2012). La importancia de la tradición oral: El grupo Coyaima - Colombia. Revista Guillermo de Ockham, 10(2), 129-143.

Rincón, A., \& Echeverría, M. C. (2000). Ciudad de territorialidades: Polémicas de Medellín. Medellín: CEHAP.

Zapata, J. (2010). Espacio y Territorio sagrado. Lógica del "ordenamiento" territorial indigena. Medellin: Universidad Nacional de Colombia. 